\title{
Complex Interplay and Hierarchy of Interactions in Two-Dimensional Supramolecular Assemblies
}

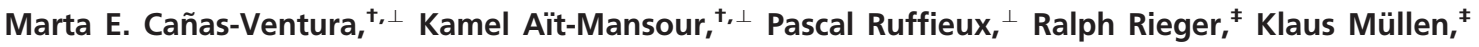 \\ Harald Brune, ${ }^{\dagger}$ and Roman Fasel ${ }^{\S, *, \perp}$ \\ ${ }^{\dagger}$ Institute of Condensed Matter Physics (ICMP), Ecole Polytechnique Fédérale de Lausanne (EPFL), Station 3, 1015 Lausanne, Switzerland, ${ }^{\ddagger}$ Max-Planck-Institut für \\ Polymerforschung, Ackermannweg 10, 55128 Mainz, Germany, ${ }^{\S}$ Department of Chemistry and Biochemistry, University of Bern, Freiestrasse 3, 3012 Bern, Switzerland, \\ and ${ }^{\perp}$ Empa, Swiss Federal Laboratories for Materials Science and Technology, nanotech@surfaces Laboratory, Feuerwerkerstrasse 39, 3602 Thun, Switzerland. "Present \\ address: Kamerlingh Onnes Laboratory, Leiden University, P.0. Box 9504, 2300 RA Leiden, The Netherlands.
}

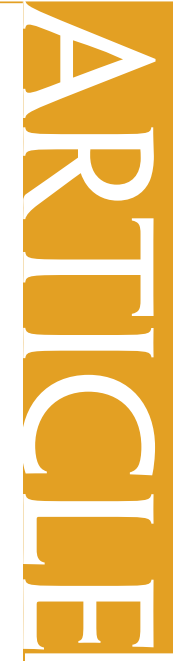

$\mathrm{T}$ he potential implementation of selfassembled supramolecular structures in molecular electronic devices asks for a profound understanding of their underlying growth mechanisms. Only their detailed knowledge will allow the reproducible fabrication of specific functional supramolecular structures for future applications. It is well established that twodimensional (2D) supramolecular order on surfaces emerges from the subtle balance of adsorbate-adsorbate and adsorbate-substrate interactions. ${ }^{1-3}$ The first can be hydrogen bonding ( $\mathrm{H}$-bonding), dipolar interactions, metal-organic coordination, or van der Waals interactions, which are always present as competing forces, while the latter are related to the corrugation of the potential energy surface seen by the molecules, such as the molecular diffusion barriers and the long-range binding energy differences on reconstructed or otherwise inhomogeneous substrates. ${ }^{4-8}$

In order to get insight into the relative strength of these interactions we have investigated mono- and bicomponent supramolecular systems with moieties allowing for a systematic and sequential increase of the number of intermolecular interaction channels. We studied the self-assembly of three closely related diaminotriazinebased molecular building blocks and a fourth complementary perylene species on $\mathrm{Au}(111)$ by means of scanning tunneling microscopy (STM). The choice of the substrate is motivated by its weak interaction with small molecules, e.g., no influence on $\mathrm{H}$-bonding between molecules, and its ( $\sqrt{ } 3$ $\times 22$ ) reconstruction $^{9}$ providing a longwavelength modulation of the molecular
ABSTRACT In order to address the interplay of hydrogen bonding, dipolar interactions, and metal coordination, we have investigated the two-dimensional mono- and bicomponent self-assembly of three closely related diaminotriazine-based molecular building blocks and a complementary perylenetetracarboxylic diimide by means of scanning tunneling microscopy. The simplest molecular species, bis-diaminotriazine-benzene, only interacts via hydrogen bonds and forms a unique supramolecular pattern on the Au(111) surface. For the two related molecular species, which exhibit in addition to hydrogen bonding also dipolar interactions and metal coordination, the number of distinct supramolecular structures increases dramatically with the number of possible interaction channels. Deposition together with the complementary perylene species, however, always results in a single well-defined supramolecular arrangement of molecules. A detailed analysis of the observed mono- and bicomponent assemblies allows shedding light on the hierarchy of the competing interactions, with important implications for the fabrication of surface-supported supramolecular networks by design.

KEYWORDS: self-assembly · hydrogen bonding - dipolar interaction - metal coordination - scanning tunneling microscopy

binding energy to the substrate emerging from the periodic stacking faults and the associated variation of the surface state charge density. ${ }^{10,11}$ The choice of the molecules is motivated as follows. The first three molecules have in common a 2,4diamino-1,3,5-triazine (DAT) group attached to a benzene core (see Scheme $1 \mathrm{a}-\mathrm{c}$ and Supporting Information for synthesis). In the para position, there is either another DAT group leading to 1,4-bis-DAT-benzene (BDATB), a symmetric molecule with a vanishing dipole moment, or a hydrogen atom leading to 6-phenyl-DAT (PhDAT) with a dipole moment of 1.2 D, or a cyano group giving rise to 6-(4'-cyanophenyl)-DAT (CPhDAT) with a large dipole moment of $4.9 \mathrm{D}$. The dipole moments have been obtained with the semiempirical Hartree-Fock Austin Model 1 (AM1).

DAT groups are known to show $\mathrm{H}$-bonding in three-dimensional (3D) $)^{12-20}$
*Address correspondence to
roman.fasel@empa.ch.

Received for review August 26, 2010 and accepted December 10, 2010.

Published online December 27, 2010. $10.1021 / \mathrm{nn} 102164 \mathrm{~g}$

() 2011 American Chemical Society 

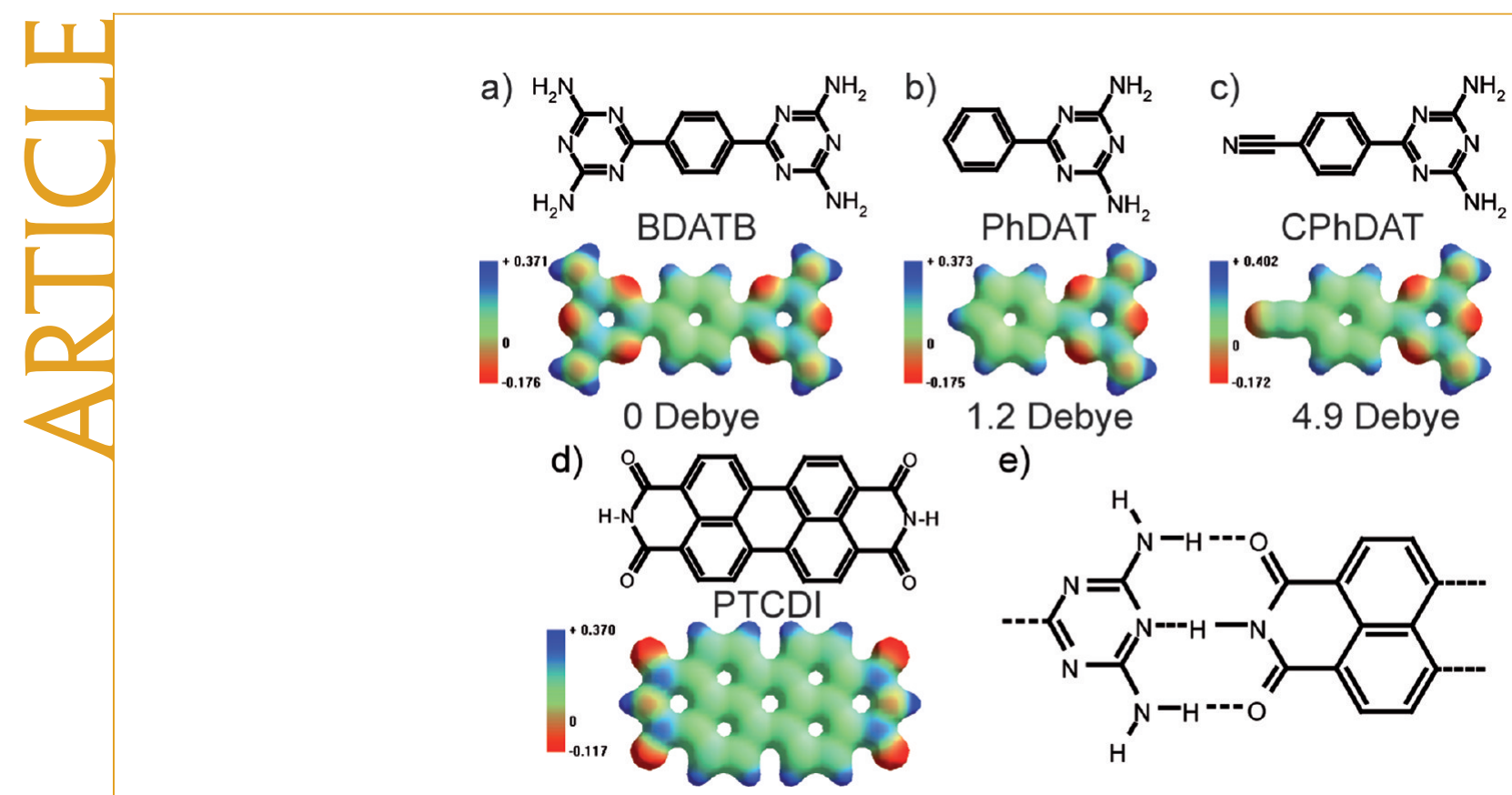

e)

Scheme 1. Skeletal Formulae of the Molecular Structures of: (a) BDATB, (b) PhDAT, (c) CPhDAT, and (d) PTCDI Molecules and (e) Schematics of the Three-Fold H-Bonding between the Complementary End-Groups DAT (common to BDATB, PhDAT, and CPhDAT) and Dicarboxylic-Imide of PTCDI ${ }^{a}{ }^{a}$ Below each structure formula we show the total charge density distribution together with the electrostatic potential due to the electronic charge distribution displayed as color (in units of e/ $\mathrm{a}_{0}$, with e being the electron charge and $\mathrm{a}_{0}$ the Bohr radius). The indicated values of the respective dipole moments have been calculated at the AM1 level of theory.

and 2D supramolecular chemistry. ${ }^{21,22}$ Accordingly, the DAT groups of the first three molecular species used here are expected to form intermolecular H-bonds. Their varying dipole moments enable one to address the competition between these $\mathrm{H}$-bonds and electrostatic interactions of varying strength. For CPhDAT, these two interactions are further complemented by the propensity of the cyano group to undergo metal coordination. $^{23-26,7}$ The three molecular species BDATB, PhDAT, and CPhDAT thus offer the possibility to explore in homomolecular assemblies the interplay and competition of H-bonding, dipole-dipole interactions, and metal coordination.

A further degree of complexity is added by studying binary molecular systems (and also ternary ones that are shown in the Supporting Information). A molecule exhibiting perfectly complementary end groups to the DAT moieties of BDATB, PhDAT, and CPhDAT is 3,4,9,10-perylenetetracarboxylic diimide (PTCDI) (Scheme 1d). Its dicarboxylic-imide moieties $(-\mathrm{CO}-\mathrm{NH}-\mathrm{CO}-$ ) are expected to form three-fold $\mathrm{H}$-bonds with the DAT moieties (Scheme 1e). In addition, this interaction is selective. The dicarboxylic-imide moiety (- $\mathrm{CO}-\mathrm{NH}-\mathrm{CO}-$ ) exhibits an $\mathrm{NH} \mathrm{H}$-bond donor (D) and two $\mathrm{CO} H$-bond acceptors $(A)$, giving rise to the well-known $\mathrm{A}-\mathrm{D}-\mathrm{A}$ sequence, ${ }^{27-30}$ while the DAT moiety $(\mathrm{NH}-\mathrm{N}-\mathrm{NH})$ has the complementary $\mathrm{D}-\mathrm{A}-\mathrm{D}$ $\mathrm{H}$-bonding sequence (Scheme 1e).

Selective DAT-dicarboxylic-imide $\mathrm{H}$-bonding has successfully been used for the formation of tapes, rosettes, and capsules in the solution phase and more recently also on surfaces. ${ }^{31-41}$ Furthermore, PTCDI is a commercially available photochemically stable dye molecule whose derivatives are among the most promising organic materials for the fabrication of organic electronic devices, like diodes, photodetectors, and field-effect transistors. This motivated extensive studies of the growth of PTCDI thin films on metal substrates. Furthermore, STM studies have been performed for PTCDI molecules adsorbed on graphite, $\mathrm{MoS}_{2}$, $\mathrm{H}$-terminated $\mathrm{Si}(111), \mathrm{Pt}(100)$, and finally on $\mathrm{Ag}-\mathrm{Si}(111)(\sqrt{ } 3 \times \sqrt{ } 3) \mathrm{R} 30^{\circ} .{ }^{42-45}$

Here we show that an increasing number of possible interaction channels leads to a higher number of distinct structures for the self-assembly of single components. While the apolar BDATB species forms a single supramolecular pattern, the polar molecules PhDAT and CPhDAT assemble into several rather different and complex supramolecular structures. In the case of $\mathbf{C P h}$ DAT one of the structures also involves metal-organic bonds. In the bimolecular systems combining any one of these molecules with PTCDI we find the anticipated three-fold $\mathrm{H}$-bonds between the complementary end groups. This leads to strongly bound heteromolecular pairs (BDATB-PTCDI) and trimers (PhDAT-PTCDIPhDAT, CPhDAT-PTCDI-CPhDAT), and these units form a single motif for each combination.

For each of the observed patterns the lowtemperature (LT) STM results are compared to calculations of the interaction energies identifying the most favorable gas-phase configurations and eventually leading to structural models. The strength and the role of $\mathrm{H}$-bonds and dipole-dipole as well as metal-organic interaction energies are compared enabling to establish hierarchies of the intermolecular interaction channels. 


\section{RESULTS AND DISCUSSION}

In the main manuscript we focus on the 2D monoand bimolecular structures and interaction hierarchies, while complementary STM data as well as detailed information on one-dimensional (1D) structures are presented in the Supporting Information.

Monocomponent Supramolecular Assemblies. This section presents the results obtained on the self-assembly of BDATB, PhDAT, respectively CPhDAT on Au(111).

BDATB on Au(111): Hydrogen Bonding. The BDATB molecules form a single close-packed structure with large domains, as can be seen in Figure 1. Along the molecular axes, this structure is stabilized by two head-to-tail $\mathrm{H}$-bonds between opposite DAT groups of neighboring molecules. In the perpendicular direction there are two lateral $\mathrm{H}$-bonds at both sides of this $C_{2 v}$ symmetric molecule. These lateral bonds are highlighted in red in the proposed structure models shown on the righthand side of Figure 1. According to their structure, two opposite DAT groups have to be displaced laterally by one-half of the molecular width in order for the headto-tail $\mathrm{H}$-bonds to form. This gives rise to two rotational domains, one where this shift is to the left and one where it is to the right. One of each domain is shown on the upper and lower part on the right-hand side of Figure 1. The molecular axis is tilted by $\pm(10 \pm 2)^{\circ}$ with respect to the unit vector $\mathbf{v}_{1}$, which is oriented along the close-packed atomic substrate rows, i.e., the crystallographic [1-10] directions of the Au(111) surface. By averaging the intermolecular distances extracted from several STM images we derive unit vectors $\mathbf{v}_{1}=(14.5 \pm$ $0.2)$ and $\mathbf{v}_{2}=(10.1 \pm 0.2) \AA$, including an angle of (50 $\pm 2)^{\circ}$. The $\mathrm{N}-\mathrm{H} \cdots \mathrm{N}$ distances are estimated to $(3.1 \pm$ $0.2)$ and (3.3 \pm 0.2$) \AA$ for the head-to-tail bonds and for the lateral ones, respectively. The molecular arrangement reported here for BDATB on Au(111) is in agreement with the one observed on $\mathrm{Au}(11,12,12){ }^{46,47}$

At rotational domain boundaries, close to steps or points defects, the BDATB molecules may adopt a slightly modified structure with respect to the one described above. For submonolayer coverages, the molecules organize into islands of the structure reported above for a full monolayer (ML).

The molecular arrangement within a row, as determined from STM images, is a shifted head-to-tail arrangement that corresponds to the lowest-energy configuration of a molecular dimer in the gas phase (Conf. 0 in Table 1) identified in our AM1-AMBER calculations (refer to Methods Section for more details). The two lateral $\mathrm{H}$-bonds are found in Conf. 52. Since the cluster size of the calculations has been limited to two molecules, they cannot address the realized combination of these two configurations. However, this arrangement appears to exhibit the lowest energy for a close-packed structure, which does not allow for the other two configurations shown in Table 1. In general, the ranking of binding energies of the various dimer configurations

www.acsnano.org

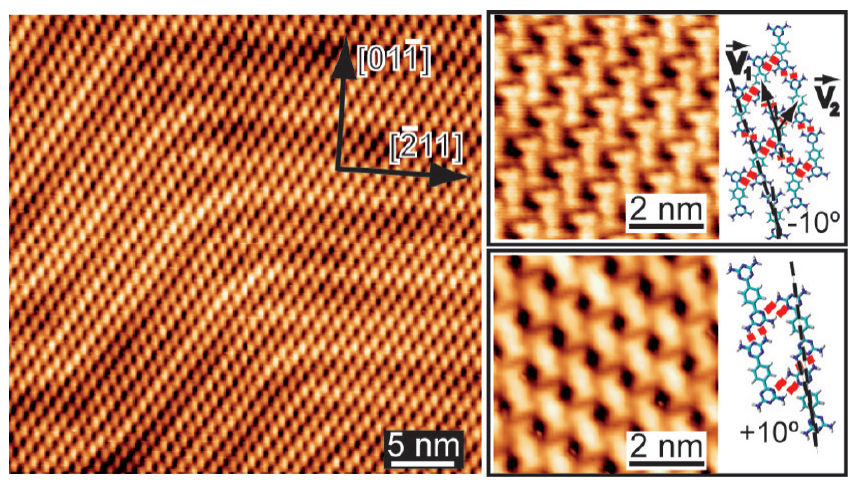

Figure 1. STM images of the supramolecular structure formed by BDATB on $\mathrm{Au}(111)$. Left: Large-scale image showing a single molecular domain. The high-symmetry directions of the underlying surface are indicated $\left(38 \times 38 \mathrm{~nm}^{2}, V_{\mathrm{t}}=-1.9 \mathrm{~V}, \mathrm{I}_{\mathrm{t}}=0.06 \mathrm{nA}\right)$. Upper right: STM image and structure model with primitive vectors $\mathbf{v}_{1}$ and $\mathbf{v}_{2}$ of superstructure unit cell; the $\mathrm{H}$-bonds are indicated by red solid lines $(5.5 \times$ $5.5 \mathrm{~nm}^{2}, V_{\mathrm{t}}=1.55 \mathrm{~V}$, and $I_{\mathrm{t}}=0.025 \mathrm{nA}$ ). Lower right: STM image of the second of the two rotational domains $\left(5.5 \times 5.5 \mathrm{~nm}^{2}, V_{\mathrm{t}}=-0.9 \mathrm{~V}\right.$, and $I_{\mathrm{t}}=0.05 \mathrm{nA}$ ).

obtained from our cluster calculations has to be combined with the energy differences resulting from steric hindrance in order to derive the extended 2D network structures. In the present case, rows are close packed and stabilized by additional inter-row $\mathrm{H}$-bonds and maximized van der Waals interaction between the molecules.

A general observation within Table 1 is that the configuration exhibiting two frontal $\mathrm{H}$-bonds between DAT groups is the most stable one for all three homomolecular dimers. In the second stable structure the molecules are oriented almost perpendicular to each other, forming an L-shaped DAT H-bond pattern (see Confs. 11, 16, and 12 for BDATB $_{2}, \mathbf{P h D A T}_{2}$, and $\mathbf{C P h D A T}_{2}$, respectively). For BDATB and PhDAT dimers the next stable configuration is a lateral DAT $\mathrm{H}$-bond pattern (Confs. 52 for BDATB $_{2}$ and 40 for PhDAT $_{2}$ ). In contrast, CPhDAT dimers prefer the antiparallel Conf. 43 as the third stable one, which is followed by the lateral DAT $\mathrm{H}$-bond pattern (Conf. 67). Conf. 43 enables the formation of $\mathrm{H}$-bonds between cyano and amine groups, and it also optimizes dipole-dipole interactions.

PhDAT on Au(111): Hydrogen Bonding and Weak Dipolar Interactions. The combination of $\mathrm{H}$-bonding and dipole-dipole interactions realized by PhDAT on $\mathrm{Au}(111)$ gives rise to a sequence of structures with increasing coverage: circular clusters of different size and spacing, 1D chains, and two close-packed structures emerging from both motives. In the Supporting Information we present a detailed discussion of all structures found. Here we focus on representative examples of 2D structures highlighting their main building principles. All structures reported are independent of deposition temperatures between 280 and $375 \mathrm{~K}$, thus they are not kinetically limited, and the most important parameter defining the resulting structure is the coverage.

Figure 2a presents the structure formed by $0.3 \mathrm{ML}$ PhDAT on Au(111). The most striking feature is the for- 


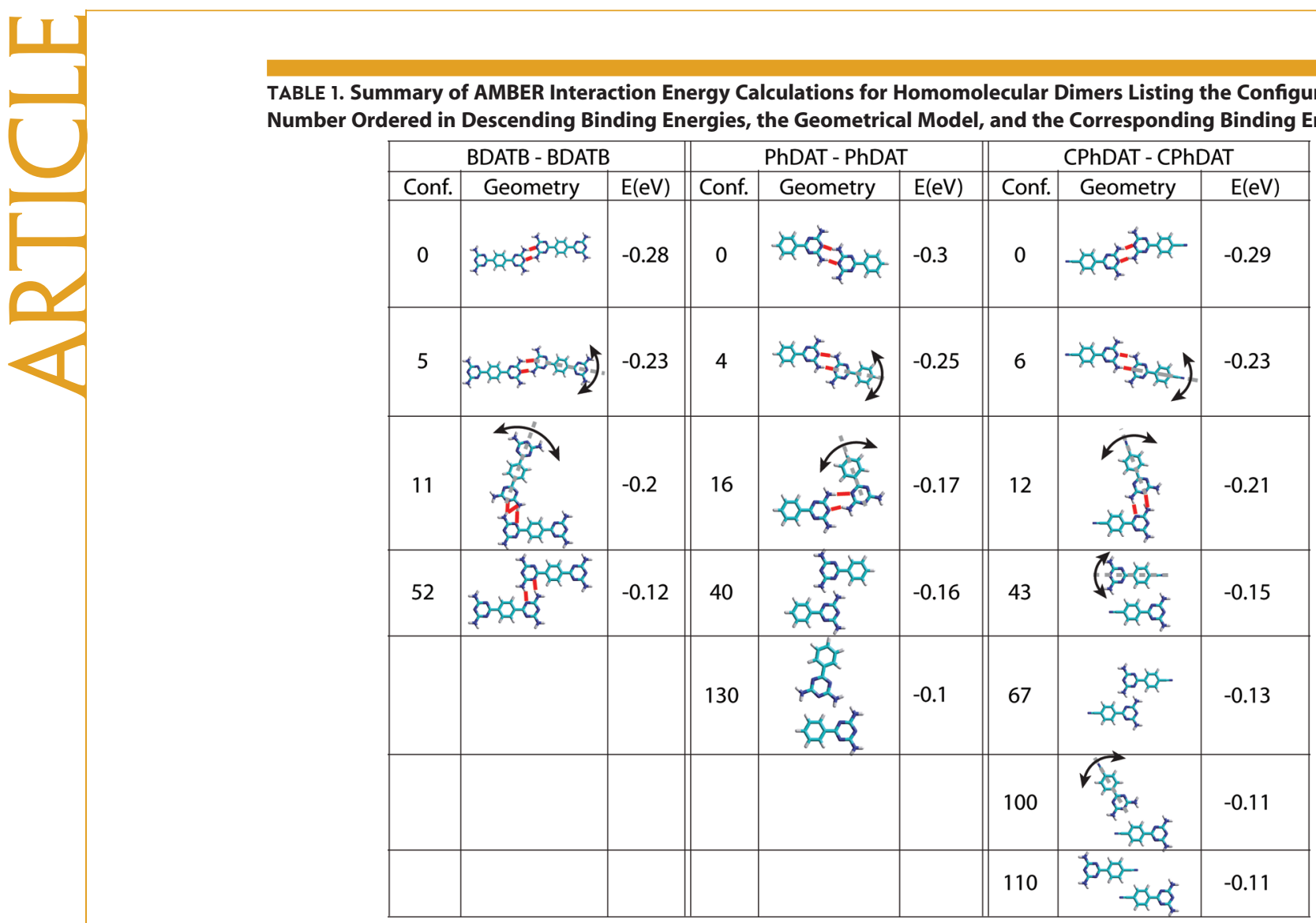

${ }^{a}$ Black curved arrows together with dashed grey lines show geometrical variations without significant energy cost. Solid red lines indicate H-bonds. More H-bonds might form depending on the case-by-case distances and precise rotation. Configurations not shown but very close in energy to a particular local minimum are marked by a curved arrow and a dashed line indicating by which rotation or translation the other energetically close configurations are obtained.

mation of regularly spaced small molecular clusters consisting of six PhDAT molecules with a flower-like appearance (6-PhDAT flower). As seen in the inset, the clusters are preferentially adsorbed on the fcc stacking areas of the $\mathrm{Au}(111)-(\sqrt{ } 3 \times 22)$ reconstruction. From high-resolution STM data, such as shown in Figure 2b, we derive that the molecules have their DAT groups oriented toward the cluster center and are linked between each other by H-bonds, as shown in the model, very similar to the $\mathrm{H}$-bonds in dimer Conf. 16 (L-dimer, Table 1).

This structure allows for two molecular tilts giving rise to clockwise and anticlockwise cluster chirality, as becomes evident when comparing the left and right side of Figure $2 \mathrm{~b}$. Both chiralities form with equal probability. While a single PhDAT molecule adsorbed on the surface is achiral, a PhDAT L-dimer is chiral. The observed formation of enantiomers of supramolecular clusters might be the result of a chiral recognition of L-dimers that spontaneously resolves the initial racemic mixture into these homochiral structures. ${ }^{48-51}$ Based on calculations, dimers in a linear arrangement (Conf. 0) are more favorable than the L-shaped ones (Conf. 16). Nevertheless, the interplay of all interactions leads to structures that are mainly based on the L-shape dimer. Structures with L-dimers are favored by the possibility to form further bonds with more molecules and the possibility to align the molecular axis with the high symmetry directions of the substrate as well as the fact that this molecular cluster arrangement compensates the dipole moments and accounts for the six-fold symmetry of the topmost atomic surface layer.

Due to the specific orientation of the molecular dipole moments, the cluster perimeters are negatively charged giving rise to mutual electrostatic cluster repulsion, which explains the regular and coveragedependent cluster spacing. Up to $0.5 \mathrm{ML}$, all clusters are situated in the fcc stacking areas. Beyond this coverage $(0.5<\theta<0.7 \mathrm{ML})$ two types of chains form on the hcp regions (see Figure $2 S$, Supporting Information), while the clusters on the fcc areas stay intact but get compressed into dense chains. The first nuclei of the hcp chains are observed at $0.4 \mathrm{ML}$, they become larger and stable at $0.5 \mathrm{ML}$. The L-motive of one of these chain-types also appears in one of the close-packed structures to be described below (see Figure 3S, Supporting Information, for the evolution of all structures with increasing coverage).

Hexagonal close packing of 6-PhDAT flower-type clusters is observed at coverages above $0.7 \mathrm{ML}$, see Figure 3a. This adsorption phase forms large homochiral domains, and we have observed both of them. The existence of extended domains of one handedness can be rationalized by the space-filling principle since clusters 


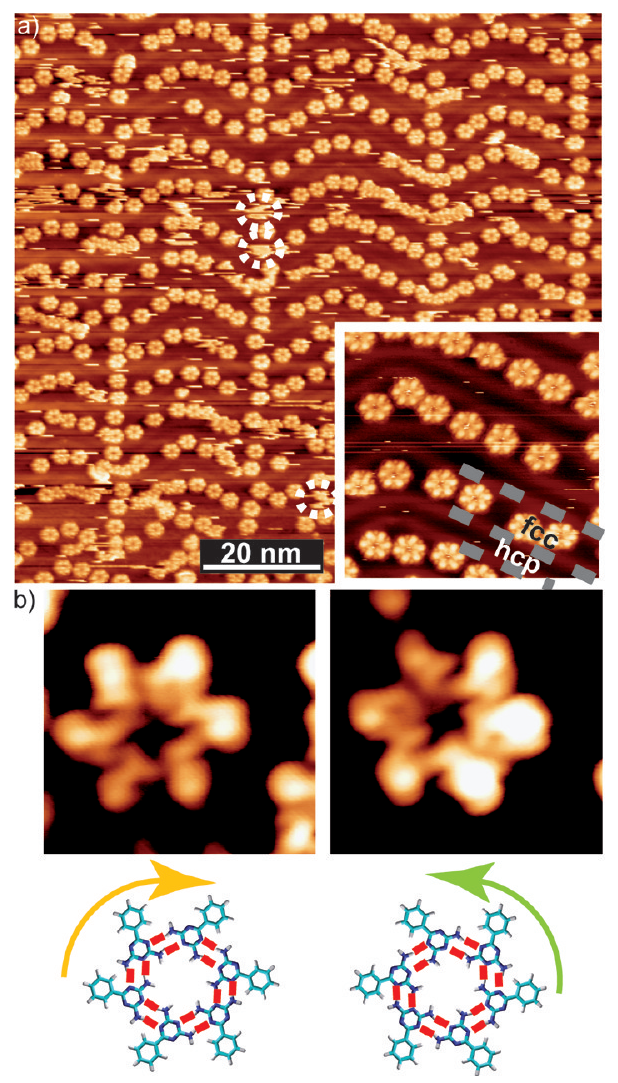

Figure 2. Regularly spaced and equally sized circular clusters formed by $0.30 \mathrm{ML}$ PhDAT on Au(111). (a) Flower-like clusters adsorbed on the fcc regions of the herringbone reconstruction $\left(100 \times 100 \mathrm{~nm}^{2}, V_{\mathrm{t}}=-1.8 \mathrm{~V}\right.$, and $\left.I_{\mathrm{t}}=0.07 \mathrm{nA}\right)$. Dotted circles highlight mobile clusters appearing fuzzy. Inset: Detail of the clusters and the partial surface dislocations (gray dashed lines) separating hcp from fcc stacking areas of the $\mathrm{Au}(111)-(\sqrt{ } 3 \times 22)$ reconstruction $\left(22 \times 22 \mathrm{~nm}^{2}, V_{\mathrm{t}}=\right.$ $-2.3 \mathrm{~V}$, and $I_{\mathrm{t}}=0.07 \mathrm{nA}$ ). (b) High-resolution STM images and corresponding models of (left) 6-PhDAT flower-type clockwise cluster $\left(3.6 \times 3.6 \mathrm{~nm}^{2}, V_{\mathrm{t}}=1.0 \mathrm{~V}\right.$, and $I_{\mathrm{t}}=0.08$ $\mathrm{nA}$ ) and (right) 6-PhDAT flower-type anticlockwise cluster $\left(4.1 \times 4.1 \mathrm{~nm}^{2}, V_{\mathrm{t}}=1.0 \mathrm{~V}\right.$, and $\left.I_{\mathrm{t}}=0.08 \mathrm{nA}\right)$. Red lines indicate the proposed $\mathrm{H}$-bonds.

of the same handedness fit more closely together. However, it implies that clusters with opposite handedness have to separate and diffuse over distances comparable with the domain size. Alternatively, they could also invert their handedness while the cluster layer gets compressed..$^{52-56}$ In either case, the clusters have to diffuse over the partial surface dislocations since in the close-packed structure they occupy both stacking areas. This implies that the interaction energies of the cyclic $\mathrm{H}$-bonding pattern in the clusters are stronger than the binding energy differences of the individual molecules between the different stacking areas of the $\mathrm{Au}(111)$ reconstruction, allowing the clusters to diffuse as intact entities. This is corroborated by the fuzzy features in STM images (Figure 2a), which we interpret as mobile 6-PhDAT clusters. An alternative scenario would be that the close packing of 6-PhDAT flower-type clusters does not arise from already formed flower-type clusters but in a molecule-by-molecule fashion. a)
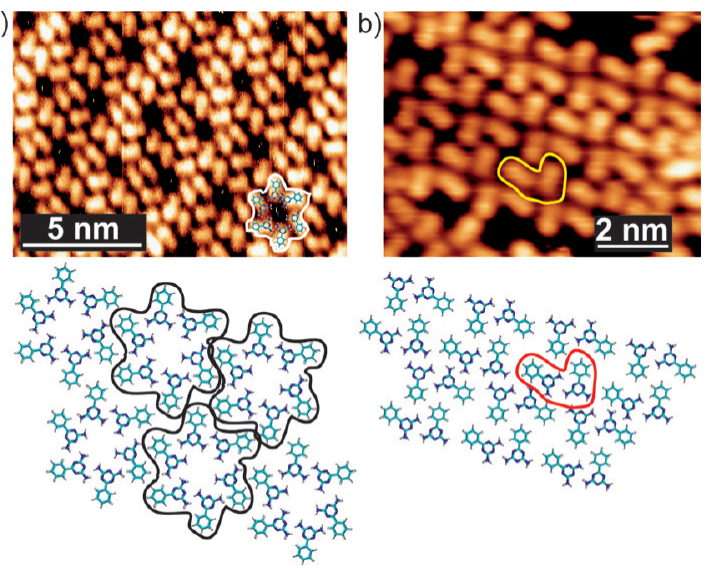

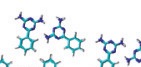

the

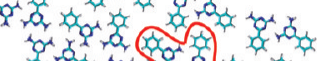

the

(a)

Prot oxt (n)

Figure 3. STM images and structure models of two closepacked adsorption phases formed by PhDAT on Au(111) for coverages above $0.7 \mathrm{ML}$. (a) Structure based on 6-PhDAT clusters, whose circumference has been marked by flowershaped white and black lines in STM image and model, respectively $\left(14 \times 8 \mathrm{~nm}^{2}, V_{\mathrm{t}}=1.8 \mathrm{~V}\right.$, and $\left.I_{\mathrm{t}}=0.1 \mathrm{nA}\right)$. (b) Close packing of L-type chains, as indicated by yellow and red lines in the STM image and the model, respectively $(9 \times 6$ $\mathrm{nm}^{2}, V_{\mathrm{t}}=1.8 \mathrm{~V}$, and $I_{\mathrm{t}}=0.1 \mathrm{nA}$ ).

A second type of densely packed phase coexists with the first and is shown in Figure 3b. It derives from L-type chains, which are based on a mixture of three dimer configurations stabilized by $\mathrm{H}$-bonds (Confs. 0 , 16, and 40, see Table 1). Two inverted L's form a unit compensating their dipole moments.

Both motives, the flower clusters and the pairs of inverted L's, consist of similar H-bonded dimer building blocks. At low coverage the interaction with the substrate decides which of them forms, since each motive is restricted to its preferred substrate stacking area. Only with increasing coverage the interactions between the motives become sufficiently large such that one of the two structures can extend over both stacking areas.

In order to rationalize the observation of stable 6-PhDAT flower-type clusters (see Figure 2 and Figure 1Sa, Supporting Information) and of other, less abundant cluster configurations (Figures $1 \mathrm{Sb}-\mathrm{d}$, Supporting Information), we calculated the binding energies of the most relevant PhDAT clusters. We determined the most stable cluster geometries by arranging the molecules of a cluster of size $n$ at the vertices of an $n$-sided polygon and by orienting them in $C_{n}$ symmetry. The polygon radius and the orientation of the long
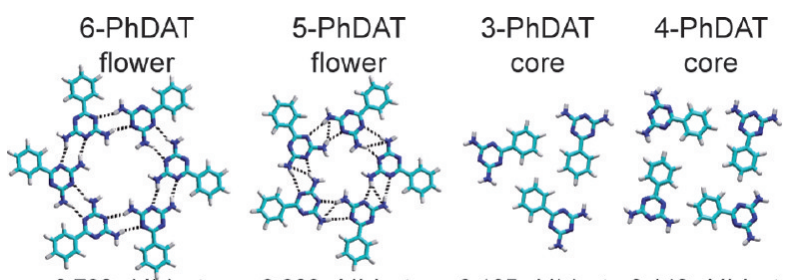

$-0.732 \mathrm{eV} /$ cluster $\quad-0.660 \mathrm{eV} /$ cluster $\quad-0.105 \mathrm{eV} /$ clust. $-0.148 \mathrm{eV} /$ clust

Figure 4. Most stable configurations determined from interaction energy calculations for regular clusters consisting of different numbers of PhDAT molecules. For 3- and 4-PhDAT clusters, the most stable configurations are the ones with DAT groups pointing away from the center. 


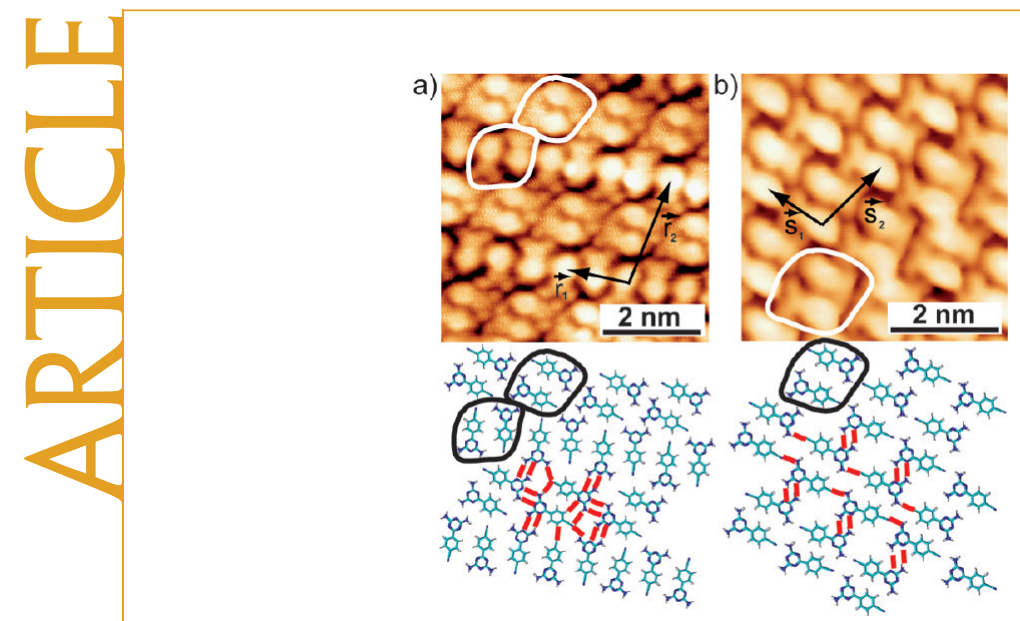

Figure 5. STM images showing the close-packed assemblies of CPhDAT on Au(111) and the corresponding structure models. The building blocks are antiparallel molecular dimers highlighted by black lines, and red bars indicate the proposed intermolecular $\mathrm{H}$-bonding. (a) Perpendicular-type close-packed structure $\left(5.5 \times 5.5 \mathrm{~nm}^{2}, V_{\mathrm{t}}=-2.0 \mathrm{~V}\right.$, and $l_{\mathrm{t}}=$ $0.2 \mathrm{nA}$ ) with unit-cell vectors $\mathbf{r}_{1}$ and $\mathbf{r}_{2}$. (b) Parallel-type closepacked structure $\left(5 \times 5 \mathrm{~nm}^{2}, V_{\mathrm{t}}=-2.0 \mathrm{~V}\right.$, and $\left.I_{\mathrm{t}}=0.1 \mathrm{nA}\right)$ with unit-cell vectors $\boldsymbol{s}_{1}$ and $\boldsymbol{s}_{2}$.

molecular axes were then varied on a dense grid of values. The most stable geometries are shown in Figure 4.

Clusters with six and five PhDAT molecules exhibit interaction energies of -732 and $-660 \mathrm{meV} / \mathrm{cluster}$, respectively. Black dotted lines in Figure 4 indicate cyclic $\mathrm{H}$-bond formation between DAT groups of neighboring molecules. Three and four molecule clusters have a structure allowing for the formation of $\mathrm{H}$-bonds around them and build the core of larger clusters. Their binding energies are -105 for 3-PhDAT and $-148 \mathrm{meV} /$ cluster for 4-PhDAT clusters, significantly lower than the ones of flower-shaped clusters.
We conclude that the interplay of $\mathrm{H}$-bonding and dipole interactions gives rise to two motives with similar intermolecular binding energies: clusters and $1 \mathrm{D}$ chains. The difference between the energies of the two motives is smaller than the binding energy difference of them to the two stacking areas of the reconstruction such that the substrate selects which of the two forms grows in its respective stacking area.

CPhDAT on Au(111): Hydrogen Bonding, Dipolar Interactions, and Metal Coordination. Among the three DAT-containing molecules, the largest structural variety upon self-assembly arises from CPhDAT. The main differences with respect to the other two molecular species are the cyano end group and the rather high dipole moment. Cyano functional groups have successfully been employed for $2 \mathrm{D}$ supramolecular engineering, ${ }^{57,58,23-26,7}$ and the dipole moment, if not fully compensated in molecular subunits, is expected to give rise to significant electrostatic interaction energies. The structures formed by CPhDAT on $\mathrm{Au}(111)$ include two types of chains (see Figures $4 S-6 S$, Supporting Information), two different close-packed structures (Figure 5), and a large number of distinct open network geometries (Figure 6 and Figure 75, Supporting Information). Large-scale overview STM images (Figures $3 S$ and 75 , Supporting Information) show the coexistence of several structures and reveal the complexity.

The STM images in Figure 5 show the two closepacked structures. Their common building block is a pair of antiparallel molecules. This arrangement optimizes the attractive dipole interactions, allows for additional H-bonds (Conf. 43 in Table 1), and compensates the overall dipole. The common feature in both closepacked structures is that these building blocks form chains oriented along $\boldsymbol{r}_{1}$ and $\boldsymbol{s}_{1}$ as indicated in Figure 5a
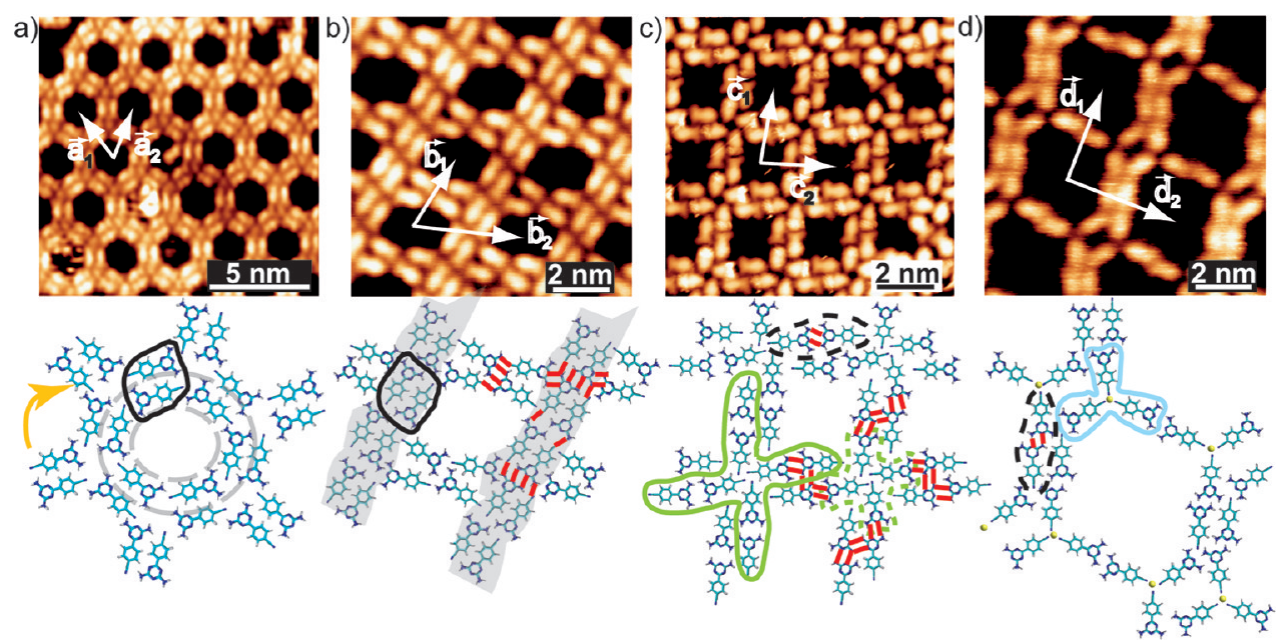

Figure 6. STM images and structure models of the open network structures formed by CPhDAT on Au(111). The building blocks are either antiparallel dimers, highlighted by full black lines, or head-to-head dimers marked by dotted black lines. Red bars indicate H-bonds. (a) Honeycomb network with clockwise helicity $\left(14 \times 14 \mathrm{~nm}^{2}, V_{\mathrm{t}}=0.8 \mathrm{~V}\right.$, and $\left.I_{\mathrm{t}}=0.01 \mathrm{nA}\right)$. (b) Rectangular network $\left(10 \times 10 \mathrm{~nm}^{2}, V_{\mathrm{t}}=-1.2 \mathrm{~V}\right.$, and $\left.I_{\mathrm{t}}=0.01 \mathrm{nA}\right)$. Gray shadows highlight the chain substructure. (c) Square open network $\left(10 \times 10 \mathrm{~nm}^{2}, V_{\mathrm{t}}=1.8 \mathrm{~V}\right.$, and $\left.I_{\mathrm{t}}=0.02 \mathrm{nA}\right)$. A full green line surrounds the repetitive cross motif and a dashed one surrounds the four molecules forming the nodal point. (d) Large open network involving three-fold cyano-Au coordination units indicated by the blue full line $\left(10 \times 10 \mathrm{~nm}^{2}, V_{\mathrm{t}}=0.9 \mathrm{~V}\right.$, and $\left.l_{\mathrm{t}}=0.01 \mathrm{nA}\right)$. 
and $b$, respectively. The structures differ by the way the chains are built and arranged with respect to each other. The structure on the right consists of only one chain type in which the molecular axes are almost parallel to the chain direction. Evidently, in this way the building blocks are parallel to each other between adjacent chains. The structure on the left implies two chain types, one with the molecular axes slightly more tilted with respect to the chain direction, but still close to parallel, and a second with the molecular axes perpendicular to the chain direction. Between adjacent chains the molecular axes of the building blocks are almost perpendicular to each other. As illustrated in the model, this relative orientation of the building blocks allows for a higher number of $\mathrm{H}$-bonds, while the parallel structure allows for a better optimization of dipole-dipole interactions. Both close-packed structures form extended domains. In the entire field of view of the STM we see either one or the other structure, from which we conclude that depending on the exact sample preparation conditions either one or the other structure is formed selectively. Deposits of CPhDAT at substrate temperatures of up to $320 \mathrm{~K}$ result in the "perpendicular" structure (Figure 5a), while the "parallel" structure (Figure 5b) is formed upon annealing of LT deposits. This implies that both structures have comparable thermodynamic stability but are separated by large kinetic barriers in agreement with the observation that the two close-packed structures never coexist.

Figure 6 presents STM images and corresponding models for the open network structures formed by CPhDAT on Au(111). The honeycomb structure shown in Figure $6 a$ is based on antiparallel CPhDAT dimers as in the close-packed structures discussed above. The cavity perimeter is formed by a circle of six CPhDAT molecules in a head-to-tail arrangement with $\mathrm{H}$-bonds between the cyano and DAT groups and with tangential molecular axes. Similar head-to-tail $\mathrm{H}$-bonding takes place in straight chains (see Figure 4Sb, Supporting Information). AMBER interaction energy calculations for this quasicircular configuration yield a binding energy of $35 \mathrm{meV}$ per molecule. Additional stabilizing factors are the overall dipole compensation in one such ring and the possibility of connecting with the adjacent honeycombs by antiparallel dimers, as shown in the model. In order for this to be possible an enantiomeric selection has to take place since all rings in one domain must have either clockwise or anticlockwise molecular orientation.

Figure $6 \mathrm{~b}$ shows a rhombic porous lattice that is composed of parallel zigzag-type chains (see Figure 4Sa, Supporting Information) linked by groups of four CPhDAT molecules oriented almost perpendicular to the chain. Also here, all CPhDAT molecules are paired into antiparallel dimers.

The square porous lattice shown in Figure $6 \mathrm{c}$ is formed by a repetition of crosses, highlighted in the

www.acsnano.org model by a full green line. Each of the four branches is a tail-to-tail CPhDAT dimer corresponding to Conf. 6. The nodal point is stabilized by electrostatic interactions between $\mathrm{N}$ atoms pointing to $\mathrm{C}$ atoms of the $\mathrm{cy}$ ano groups of neighboring molecules. This node configuration has been highlighted in the model by a dashed green line and has an interaction energy of $37 \mathrm{meV}$ per molecule, according to our AMBER calculations. The crosses are linked among each other by lateral $\mathrm{H}$-bonds.

A forth type of porous lattice is shown in Figure $6 \mathrm{~d}$. This structure also involves tail-to-tail molecular dimers and forms for coverages below $0.3 \mathrm{ML}$ and upon annealing the surface after the adsorption of the molecules to above $440 \mathrm{~K}$. The fact that annealing is required and that this structure forms more often near step edges suggests the involvement of Au atoms. This is confirmed by three-fold coordination nodes clearly revealed in the STM images, where one gold adatom binds to three cyano groups. The coordination of cyano complexes to metal centers is well-known from 3D supramolecular chemistry. ${ }^{59-62}$ The cyano group has a lone electron pair that can coordinate in different geometries to heavy metal atoms. Three-fold coordination is unusual in 3D but can be stabilized in 2D, as observed for biphenolate end groups linking to Fe atoms and for dicarbonitrile end groups linking to Co atoms. $^{23-26,7}$ Here we observe yet another example of such three-fold coordination, this time for cyano groups to Au adatoms. The role of the surface in stabilizing such unusual coordination is the confinement of the molecules to a plane, the changes of the electronic structure of the molecules caused by their interaction with the substrate, and finally the hybridization of the metal adatom with the substrate that changes its electronic configuration compared to metal centers in 3D metal-organic supramolecular structures. Note that the three-fold substrate symmetry is not required to stabilize three-fold coordination. ${ }^{23}$

The four coexisting open network structures found for CPhDAT can be considered as a validation of Wuest's statement for the case of 2D self-assembly on surfaces - "When tectons can form multiple interactions that are strong and directional, such as $\mathrm{H}$-bonds, crystallization normally generates open networks". ${ }^{16}$ We carefully checked, by variation of the experimental parameters, deposition temperature and flux, postdeposition annealing temperature, and coverage whether the coexistence of these structures is caused by kinetic limitations or by their energetic degeneracy. We generally observe that open networks require a second deposition step onto a precovered surface, implying the need of a reduced molecular mobility. Which open network structure forms depends on coverage as well as on the surface temperatures during the first and second deposition steps and during postannealing. The fact that there exists a whole parameter range for which 
the structures coexist indicates that they are energetically very close.

As outlined in the Supporting Information, one can come up with a tentative energy hierarchy for the CPhDAT structures (Figure 8S, Supporting Information). Zigzag double chains created by deposition at $190 \mathrm{~K}$ transform into straight double chains upon annealing to $270 \mathrm{~K}$; these chains assemble into the parallel closepacked structure upon further annealing to $315 \mathrm{~K}$. On the other hand, direct deposition at $320 \mathrm{~K}$ gives the perpendicular close-packed structure. In the last case the diffusing species is smaller than in the first, where entire chains have to be rearranged. Therefore kinetic limitations are smaller in the last case which likely presents the global energy minimum. The zigzag chains represent local energy minima and thus kinetically stabilized metastable states. The first two open-network structures transform upon annealing and therefore clearly are kinetically limited, and the last two presumably as well since they require a second deposition step to be created; the structures formed in the first step cause the necessary kinetic limitations for the open network to form. The fact that they are stable for annealing up to $365 \mathrm{~K}$ shows that kinetic barriers separating them from the global minimum are high. To sum up, these configurations are separated by large energy barriers making them metastable and observable. These kinetic limitations enable to reach them only by a certain formation history. Annealing leads to structural modifications if these barriers can be overcome. The open network structures are close in energy and present large kinetic limitations, which make the preparation of a single open-network species difficult.

The structures formed by all three molecules can be summarized and rationalized as follows: BDATB allows for $\mathrm{H}$-bonds on both ends; therefore, parallel molecules line up in chains where adjacent molecules are laterally displaced by one-half of their width due to the D-A-D H-bonding sequence of the DAT group. In the single structure found for this molecule, these chains align parallel in such a way that two lateral $\mathrm{H}$-bonds can be established. PhDAT allows for $\mathrm{H}$-bonds only at the DAT end. These bonds are stronger than the electrostatic repulsion between alike molecular ends, and therefore, DAT ends bind together, either in circular clusters having zero dipole moment and repelling each other or in pairs of inverted Ls. The larger electrostatic dipole of CPhDAT molecules disfavors alike ends to bind to each other, and therefore, clusters cannot form. Instead, the molecules form antiparallel or tail-to-tail dimers, with vanishing overall dipole moment. Each of the molecules in the antiparallel dimers has a head-to-tail bond to the corresponding molecule of the next dimer. Corresponding chains appear not only in both dense structures but also in the first two of the open networks, where they are bent to a circle in the honeycomb network or straight and laterally con-
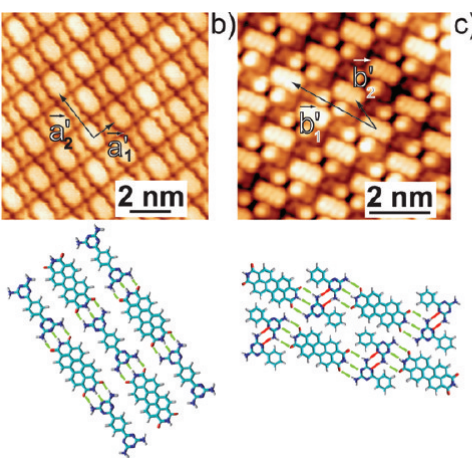

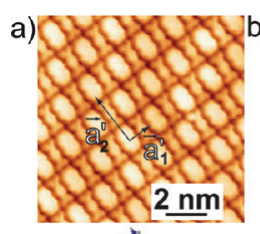

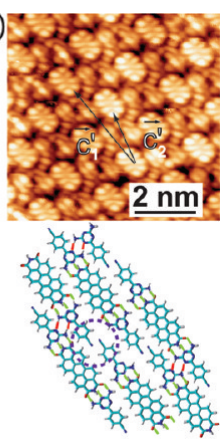

Figure 7. STM images and structure models of the bicomponent close-packed structures formed on $\mathrm{Au}(111)$. Elongated bright rounded rectangles are associated with PTCDI molecules. In the models, three-fold axial H-bonds formed by the complementary end groups are indicated by green lines and lateral ones by red lines. (a) Regular close-packed BDATB-PTCDI lattice $\left(10 \times 10 \mathrm{~nm}^{2}, V_{\mathrm{t}}=-0.3 \mathrm{~V}\right.$, and $l_{\mathrm{t}}=$ $0.05 \mathrm{nA}$ ). (b) (PhDAT) ${ }_{2}$ PTCDI lattice, intramolecular resolution identifies both molecules $\left(7 \times 7 \mathrm{~nm}^{2}, V_{\mathrm{t}}=-2.0 \mathrm{~V}\right.$, and $\left.I_{\mathrm{t}}=0.17 \mathrm{nA}\right)$. (c) $(\text { CPhDAT) })_{2}$ PTCDI lattice $\left(7 \times 7 \mathrm{~nm}^{2}, V_{\mathrm{t}}\right.$ $=-1.5 \mathrm{~V}$, and $\left.I_{\mathrm{t}}=0.1 \mathrm{nA}\right)$. A dashed circle highlights the sideby-side positioning of cyano groups.

nected by short chains of two antiparallel dimers in the rhombic network. The last two networks imply tailto-tail dimers. In the square network the polar dimer ends link in four-fold nodes while they form three-fold metal-organic bonds in the large open network.

The most striking feature comparing the three molecules is that the number of structures increases as more intermolecular interaction channels become available. If these are comparable in energy, then many structures coexist. In addition, the interaction with the substrate is not homogeneous due to the reconstruction, it also depends on the orientation of the molecules, and both may influence how the molecular dipole is screened. ${ }^{63}$ This adds complexity to the system and favors structural manifold.

Bicomponent Supramolecular Structures: Promoting ThreeFold Hydrogen Bonding in Heteromolecular Systems. In contrast to the variety of structures resulting from monocomponent deposits, heteromolecular systems driven by the selective formation of three-fold $\mathrm{H}$-bonds (see Scheme 1e) between two complementary molecular species reduce the number of distinct motives to one per combination of molecules.

Codeposition of BDATB with PTCDI onto Au(11, $12,12)$ has been reported to lead to large and wellordered domains of closely packed BDATB-PTCDI chains on the (111) terraces of the vicinal surface. ${ }^{46,47}$ As Figure 7a shows, the same structure forms on the extended terraces of $\mathrm{Au}(111)$. The PTCDI molecules appear as bright double lobes in negative bias STM images, reflecting the charge distribution of their highest occupied molecular orbital (HOMO). BDATB and PTCDI both have $C_{2 v}$ symmetry. They can form at both ends axial three-fold $\mathrm{H}$-bonds with the respective complementary molecule. This explains the BDATB-PTCDI chains which have also been reported on $\mathrm{NaCl} / \mathrm{Au}(11$, 
TABLE 2. Configurations and Binding Energies of Heterodimers Created Between the Three Molecules BDATB, CPhDAT, and PhDAT and the Molecule PTCDI ${ }^{a}$

\begin{tabular}{|c|c|c|c|c|c|c|c|c|}
\hline \multicolumn{3}{|c|}{ BDATB - PTCDI } & \multicolumn{3}{|c|}{ PhDAT - PTCDI } & \multicolumn{3}{|c|}{ CPhDAT - PTCDI } \\
\hline Conf. & Geometry & $E(e V)$ & Conf. & Geometry & $\mathrm{E}(\mathrm{eV})$ & Conf. & Geometry & $\mathrm{E}(\mathrm{eV})$ \\
\hline 0 & 证 & -0.40 & 0 & $x-0,300$ & -0.5 & 0 & $-x<00 \pi$ & -0.41 \\
\hline 3 & 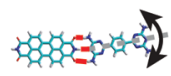 & -0.36 & 4 & & -0.33 & 2 & ; & -0.37 \\
\hline 8 & 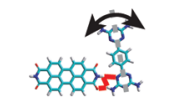 & -0.26 & 18 & & -0.26 & 14 & & -0.26 \\
\hline 21 & 038 & -0.21 & 34 & & -0.21 & 39 & & -0.2 \\
\hline 42 & & -0.18 & 56 & & -0.18 & 58 & 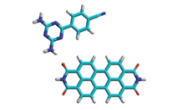 & -0.19 \\
\hline
\end{tabular}

${ }^{a}$ Red solid lines indicate H-bonds.

$12,12)^{64}$ and on $2 \mathrm{ML} \mathrm{Ag} / \mathrm{Pt}(111)-(25 \times 25) \cdot{ }^{65}$ In the close-packed structure, adjacent chains are shifted by half of a unit leading to a molecular alternation also perpendicular to the chains. From STM images taken on larger terraces we derive that: (i) the $(\sqrt{ } 3 \times 22)$ reconstruction has only negligible influence on the orientation and size of molecular domains; (ii) the reconstruction itself is unaffected by the molecular overlayer indicating weak molecule-substrate interaction, and (iii) upon annealing all domains orient their molecular chains along one of the three close-packed [1-10] directions.

PhDAT and CPhDAT molecules can form only at one end three-fold axial H-bonds with PTCDI. Figure $7 \mathrm{~b}$ and $\mathrm{c}$ shows that this leads to the anticipated PhDAT-PTCDI-PhDAT and CPhDAT-PTCDICPhDAT trimers implying 1:2 stoichiometry. For the tunneling parameters used in Figure $7 \mathrm{~b}$, the phenyl ring of the PhDAT molecules appears bright and the DAT group dark, due to the larger contribution from the $\pi$-orbitals to the tunneling current. With this contrast the STM images nicely display the successive

PhDAT-PTCDI-PhDAT units in the chains as dot-bardot sequence. The close packing of the chains and their mutual shift are rationalized by the lateral $\mathrm{H}$-bonds indicated in the model and corresponding to Conf. 40 in Table 2.

The axis of the unit formed by the three molecules is parallel to the chain direction. Table 2 summarizes the results of interaction energy calculations for the heteromolecular dimers of interest in this study, namely,

BDATB-PTCDI, PhDAT-PTCDI, and CPhDAT-PTCDI. For all three heteromolecular dimers the configuration with the anticipated three-fold frontal $\mathrm{H}$-bond is the most stable one (Conf. 0). The highest interaction energy is obtained for the PTCDI-PhDAT dimer, followed www.acsnano.org by the dimer involving CPhDAT, and, with a very small energy difference, the one where PTCDI binds to a BDATB molecule. Comparing the energies with the ones of homomolecular dimers, the preference for hetero- vs homocomplex formation is clearly evidenced. For these molecule combinations the calculations predict therefore intermixed bicomponent structures to be more stable than homomolecular phases.

In the close-packed structure formed by the CPhDAT-PTCDI-CPhDAT chains (Figure 7c), the axes of the building blocks are slightly rotated with respect to the chain direction. This tilt enables a side-byside position of the polar cyano end groups, as highlighted by a circle in the model. Further evidence for this structure comes from the lattice constant along the chains, which is significantly smaller than in a head-tohead configuration with realistic $\mathrm{H}$-bond lengths. As in the homomolecular structures discussed above, the large dipole moment of CPhDAT manifests itself by an antiparallel arrangement of the CPhDAT molecules of adjacent chains, similar to Conf. 67 reported in Table 1. The two possible tilt directions with respect to the unit cell vector $\mathbf{c}_{1}{ }^{\prime}$ give rise to the formation of two mirror domains.

With the aim of further expanding the variety of open network structures we also codeposited two of the three closely related molecules. We expected the different molecular lengths to lead to new pore sizes. However, both molecular mixtures phase separate into extended monocomponent domains. The surface morphology after codeposition of CPhDAT and BDATB is shown in Figure 10Sa, Supporting Information, for close to a full ML coverage. Pure-BDATB and -CPhDAT domains are separated by sharp boundaries (Figure 10Sb, Supporting Information). A detailed analysis reveals that the molecules form exactly the close-packed struc- 
tures reported above for monocomponent deposits. Phase separation is maintained up to annealing temperatures of, at least, 440 K. Figure 11S, Supporting Information, shows STM images of the molecular structures resulting from codeposition of the three molecular species PTCDI, BDATB, and CPhDAT. Evidently, there is no mixing into a homogeneous structure either, but phase separation takes place into bicomponent BDATB-PTCDI and CPhDAT-PTCDI superlattices with their respective structures as reported for the corresponding bimolecular deposits in Figure 7. These observations further underline the dominant role played by $\mathrm{H}$-bonding (and in particular the three-fold axial H-bonding motif between DAT and dicarboxylic-imide end groups) among the different interaction channels.

Finally, we have calculated dipole-dipole interaction energies in order to estimate their importance compared to the other competing interactions in the formation of self-assembled supramolecular structures (see the Supporting Information for details). So far only a few studies have quantified dipole-dipole interactions in supramolecular structures at surfaces $58,63,66,67$ and in the gas phase and solution, ${ }^{68,69}$ while numerous studies have been devoted to the binding energies of $\mathrm{H}$-bonds. The contribution of dipolar interactions to the total binding energy of supramolecular assemblies is in general comparatively small. However, there are many channels through which dipole interactions can contribute, such as induced dipole moments from neighboring molecules, dipoles and screening through image charges in the substrate, and substrate mediated long- and short-range interactions..$^{70-72}$ The way they precisely affect the final structure is poorly understood, and a first step is made here by assessing their part in the total binding energy.

It is generally assumed that dipole-dipole interaction energies are in the range of $0.05-0.52 \mathrm{eV}$, while $\mathrm{H}$-bonds may range from $0.04 \mathrm{up}$ to $1.5 \mathrm{eV}$. The dipole-dipole interaction energies calculated for the PhDAT-PhDAT and CPhDAT-CPhDAT dimers are all at the lower end of this range (Table 1S, Supporting Information). We note that the dipole-dipole interaction is attractive only for two particular configurations of each homomolecular dimer, while it is repulsive for the other ones (see black and red values in Table 1S, Supporting Information). Furthermore, dipolar interactions are significantly weaker than $\mathrm{H}$-bonding in all cases. Their contribution to the total binding energy amounts to a maximum of $30 \%$ and $15 \%$ for Confs. 43 and 100 of CPhDAT $_{2}$, whereas for PhDAT only $2 \%$ and $4 \%$ of the binding energy stem from dipole-interactions for Confs. 40 and 130, respectively. Even though dipole-dipole interactions might thus not play a decisive role in the final stabilization of self-assembled structures, they might very well play a role during the dynamic assembly process. For CPhDAT, for example, dipole-dipole interactions favor the formation of CPhDAT dimers with antiparallel dipole moments, a configuration that is the fundamental building block for all supramolecular structures observed, while $\mathrm{H}$-bonding interactions would favor many more stable configurations than this particular one with antiparallel dipole moments.

\section{CONCLUSIONS}

We have reported that an increasing number of intermolecular interaction channels leads to the formation of an increasing number of distinct supramolecular structures: Only a single close-packed structure is observed for BDATB interacting by $\mathrm{H}$-bonds only, whereas chains, clusters, and two different closepacked structures are observed for PhDAT, the small dipole moment of which adds electrostatic interactions to H-bonds. For CPhDAT molecules exhibiting a larger dipole moment and a cyano group enabling metal coordination, the formation of chains, four open-network structures, and two close-packed structures is observed, and a suggestion for their energetic hierarchy is derived.

We have further shown that molecular building blocks exhibiting complementary end groups promote the selective formation of heterocomplex assemblies. For our three DAT-based molecules combined with PTCDI, this leads to the formation of the anticipated three-fold axial $\mathrm{H}$-bonds between the respective complementary molecules. The strength and the directionality of such bonds promotes the growth of a single, well-ordered phase per molecular combination consisting of chains formed by building units containing two, respectively, three molecules. The single structure is in marked contrast to the multiple structures observed for single-component deposits that result from competing $\mathrm{H}$-bond, dipolar, and metal coordination interactions.

\section{METHODS}

STM experiments have been performed under ultrahigh vacuum (UHV) conditions at a base pressure below $1 \times 10^{-10}$ mbar. The $A u(111)$ surface has been prepared by repeated cycles of argon ion sputtering ( $\left.p_{\mathrm{Ar}}=2 \times 10^{-5} \mathrm{mbar}\right)$ at room temperature and subsequent annealing at $T=720 \mathrm{~K}$. BDATB, PhDAT, CPhDAT, and PTCDI molecules have been evaporated from quartz crucibles maintained at temperatures adjusted for each molecular species so as to give a deposition flux of $0.1-0.2 \mathrm{ML}$ / min. The sample temperature during deposition, possible annealing temperatures after deposition, and the molecular coverage are given for each figure in its caption and/or eluded to in the text. Two Omicron STMs have been employed, the first has been a variable-temperature one operated at $40 \mathrm{~K}$ and the second a LT one operated at $77 \mathrm{~K}$. Mechanically cut Pt/Ir wires or electrochemically etched $\mathrm{W}$ wires served as STM tips.

Interaction energy calculations for molecular dimers have been performed according to the following procedure. The ge- 
ometries of the individual molecules have been optimized using the semiempirical Hartree-Fock AM1 that is known to reproduce well the ground-state geometry of organic molecules. ${ }^{73,74}$ Interaction energy calculations were then performed without further relaxation of the individual molecules within the classical molecular mechanics scheme at the AMBER level of theory. ${ }^{75}$ Although interaction energies are often underestimated this way, energy differences between different structures still provide a valid picture of the energy hierarchy. The same approach has previously proven successful for the characterization of weak interactions in molecular adsorbate systems. ${ }^{76}$ The substrate was not explicitly considered in the calculations, but the molecules were forced to stay in one plane. Parameters considered in the calculations were the distance between the centers of the molecules and the angle between the long axes of the two molecules. For the interaction energy calculations, one of the two molecules was placed at the origin, and the second molecule was translated according to a given matrix of points. At each of these locations, the long axis of the molecule was rotated such that all possible configurations for each dimer were explored. The relative molecular orientations and distances have been varied in steps of $5^{\circ}$ and $0.2 \AA$, respectively. Then the 250 most stable configurations were ranked in order of decreasing stability, i.e., configuration number 0 (Conf. 0 ) is the ground state. An average decrease of the interaction energy by about $80 \%$ was observed within the first 250 configurations.

Acknowledgment. We gratefully acknowledge financial support from the European Commission (NMP3-CT-2004-001561 RADSAS) and the Swiss National Science Foundation.

Supporting Information Available: Molecular synthesis, additional 1D structures, complementary information for 2D mono-, bi-, and even three-component self-assembly, and dipole-dipole interaction energy calculations. This material is available free of charge via the Internet at http://pubs.acs.org.

\section{REFERENCES AND NOTES}

1. Barth, J. V. Molecular Architectonic on Metal Surfaces. Annu. Rev. Phys. Chem. 2007, 58, 375.

2. Barth, J. V.; Constantini, G.; Kern, K. Engineering Atomic and Molecular Nanostructures at Surfaces. Nature 2005, 437, 671-679.

3. Bartels, L. Tailoring Molecular Layers at Metal Surfaces. Nature Chem. 2010, 2, 87-95.

4. Yokoyama, T.; Kamikado, T.; Yokoyama, S.; Mashiko, S. Conformation Selective Assembly of Carboxyphenyl Substituted Porphyrins on Au(111). J. Chem. Phys. 2004, 121, 11993.

5. Hoster, H. E.; Roos, M.; Breitruck, A.; Meier, Ch.; Tonigold, K.; Waldmann, T.; Ziener, U.; Landfester, K.; Behm, R. J. Structure Formation in Bis(terpyridine) Derivative Adlayers: Molecule-Substrate versus Molecule-Molecule Interactions. Langmuir 2007, 23, 11570-11579.

6. Kühnle, A. Self-assembly of Organic Molecules at Metal Surfaces. Curr. Opin. Colloid Interface Sci. 2009, 14, 157-168.

7. Schlickum, U.; Decker, R.; Klappenberger, F.; Zoppellaro, G.; Klyatskaya, S.; Ruben, M.; Silanes, I.; Arnau, A.; Kern, K.; Brune, H.; Barth, J. V. Metal-Organic Honeycomb Nanomeshes with Tunable Cavity Size. Nano Lett. 2007, 7, 3813-3817.

8. Schlickum, U.; Decker, R.; Klappenberger, F.; Zoppellaro, G.; Klyatskaya, S.; Auwärter, W.; Neppl, S.; Kern, K.; Brune, H.; Ruben, M.; Barth, J. V. Chiral Kagomé Lattice from Simple Ditopic Molecular Bricks. J. Am. Chem. Soc. 2008, 130, 11778-11782.

9. Barth, J. V.; Brune, H.; Ertl, G.; Behm, R. Scanning Tunneling Microscopy Observations on the Reconstructed Au(111) Surface: Atomic Structure, Long-range Superstructure, Rotational Domains, and Surface Defects. J. Phys. Rev. B: Condens. Matter Mater. Phys. 1990, 42, 9307.

10. Chen, W.; Madhavan, V.; Jamneala, T.; Crommie, M. F.
Scanning Tunneling Microscopy Observation of an Electronic Superlattice at the Surface of Clean Gold. Phys. Rev. Lett. 1998, 80, 1469.

11. Bürgi, L.; Brune, H.; Kern, K. Imaging of Electron Potential Landscapes on Au(111). Phys. Rev. Lett. 2002, 89, 176801.

12. Cousson, A.; Nicolaï, B.; Fillaux, F. Melamine (1,3,5-triazine2,4,6-triamine): a Neutron Diffraction Study at $14 \mathrm{~K}$. Acta Crystallogr., Sect. E: Struct. Rep. Online 2005, 61, 222.

13. Gamez, P.; Reedijk, J. 1,3,5-Triazine-based Synthons in Supramolecular Chemistry. Eur. J. Inorg. Chem. 2006, 29-42.

14. Maly, K. E.; Gagnon, E.; Maris, T.; Wuest, J. D. Engineering Hydrogen-bonded Molecular Crystals Built from Derivatives of Hexaphenylbenzene and Related Compounds. J. Am. Chem. Soc. 2007, 129, 4306-4322.

15. Thalacker, C.; Würthner, F. Chiral Perylene BisimideMelamine Assemblies: Hydrogen Bond-directed Growth of Helically Stacked Dyes with Chiroptical Properties. Adv. Funct. Mater. 2002, 12, 209.

16. Malek, N.; Maris, T.; Simard, M.; Wuest, J. D. Molecular Tectonics. Selective Exchange of Cations in Porous Anionic Hydrogen-bonded Networks Built from Derivatives of Tetraphenylborate. J. Am. Chem. Soc. 2005, 127, 5910-5916.

17. Fournier, J.-H.; Maris, T.; Wuest, J. D. Molecular Tectonics. Porous Hydrogen-bonded Networks Built from Derivatives of 9,9'-spirobifluorene. J. Org. Chem. 2004, 69, 1762-1775.

18. Demers, E.; Maris, V.; Wuest, J. D. Molecular Tectonics. Porous Hydrogen-bonded Networks Built from Derivatives of 2,2',7,7' -tetraphenyl-9,9' -spirobi[9H-fluorene]. Cryst. Growth Des. 2005, 5, 1227-1235.

19. Laliberte, D.; Maris, T.; Demers, E.; Helzy, F.; Arseneault, M.; Wuest, J. D. Molecular Tectonics. Hydrogen-bonded Networks Built from Tetra- and Hexaanilines. Cryst. Growth Des. 2005, 5, 1451-1456.

20. Laliberte, D.; Maris, T.; Wuest, J. D. Molecular Tectonics. Porous Hydrogen-bonded Networks Built from Derivatives of Pentaerythrityl Tetraphenyl Ether. J. Org. Chem. 2004, 69, 1776-1787.

21. Jonkheijm, P.; Zdanowska, M.; Miura, A.; Hoeben, F. J. M.; De Feyter, S.; Schenning, A. P. H. J.; De Schryver, F. C.; Meijer, E. W. $\pi$-conjugated Oligo-(p-phenylenevinylene) Rosettes and their Tubular Self-assembly. Angew. Chem., Int. Ed. 2004, 43, 74.

22. Perdigao, L. M. A.; Perkins, E. W.; Ma, J.; Staniec, P. A.; Rogers, B. L.; Champness, N. R.; Beton, P. H. Bimolecular Networks and Supramolecular Traps on Au(111). J. Phys. Chem. B 2006, 110, 12539-12542.

23. Stepanow, S.; Lin, N.; Payer, D.; Schlickum, U.; Klappenberger, F.; Zoppellaro, G.; Ruben, M.; Brune, H.; Barth, J. V.; Kern, K. Surface-Assisted Assembly of 2D Metal-Organic Networks That Exhibit Unusual Threefold Coordination Symmetry. Angew. Chem., Int. Ed. 2007, 46, 710-713.

24. Kühne, D.; Klappenberger, F.; Decker, R.; Schlickum, U.; Brune, H.; Klyatskaya, S.; Ruben, M.; Barth, J. V. HighQuality 2D Metal-Organic Coordination Network Providing Giant Cavities within Mesoscale Domains. J. Am. Chem. Soc 2009, 131, 3881-3883.

25. Marschall, M.; Reichert, J.; Weber-Bargioni, A.; Seufert, K.; Auwärter, W.; Klyatskaya, S.; Zoppellaro, G.; Ruben, M.; Barth, J. V. Random Two-dimensional String Networks Based on Divergent Coordination Assembly. Nat. Chem. 2010, 2, 131.

26. Kühne, D.; Klappenberger, F.; Decker, R.; Schlickum, U.; Brune, H.; Klyatskaya, S.; Ruben, M.; Barth, J. V. SelfAssembly of Nanoporous Chiral Networks with Varying Symmetry from Sexiphenyl-dicarbonitrile on $\mathrm{Ag}(111)$. J. Phys. Chem. C 2009, 113, 17851.

27. Prins, L. J.; Reinhoudt, D. N.; Timmerman, P. Noncovalent Synthesis Using Hydrogen Bonding. Angew. Chem., Int. Ed. 2001, 40, 2382-2426.

28. Whitesides, G. M.; Simanek, E. E.; Mathias, J. P.; Seto, C. T.; 
Chin, D. N.; Mammen, M.; Gordon, D. M. Noncovalent Synthesis: Using Physical-organic Chemistry to Make Aggregates. Acc. Chem. Res. 1995, $28,37$.

29. Whitesides, G. M.; Mathias, J. P.; Seto, C. T. Molecular Selfassembly and Nanochemistry: a Chemical Strategy for the Synthesis of Nanostructures. Science 1991, 254, 1312.

30. Scherrington, D. C.; Taskinen, K. A. Self-assembly in Synthetic Macromolecular Systems via Multiple Hydrogen Bonding Interactions. Chem. Soc. Rev. 2001, 30, 83.

31. Zerkowski, J. A.; Mathias, J. P.; Whitesides, G. M. Design of Organic Structures in the Solid State: Molecular Tapes Based on the Network of Hydrogen Bonds Present in the Cyanuric Acid - Melamine Complex. J. Am. Chem. Soc 1994, 116, 4305.

32. Mascal, M.; Hansen, J.; Fallon, P. S.; Blake, A. J.; Heywood, B. R.; Moore, M. H.; Turkenburg, J. P. From Molecular Ribbons to a Molecular Fabric. Chem.—Eur. J. 1999, 5, 381

33. Lehn, J.-M.; Mascal, M.; DeCian, V.; Fischer, J. Molecular Recognition Directed Self-assembly of Ordered Supramolecular Strands by Cocrystallization of Complementary Molecular Components. J. Chem. Soc., Chem. Commun. 1990, 6, 479.

34. Zerkowski, J. A.; Seto, C. T.; Whitesides, G. M. Solid-state Structures of "Rosette" and "crinckled tape" Motifs Derived from the Cyanuric Acid-melamine Lattice. J. Am. Chem. Soc 1992, 114, 5473.

35. Mathias, J. P.; Simanek, E. E.; Zerkowski, J. A.; Seto, C. T.; Whitesides, G. M. Structural Preferences of Hydrogenbonded Networks in Organic Solution - the Cyclic $\mathrm{CA}_{3} \cdot \mathrm{M}_{3}$ “Rosette. J. Am. Chem. Soc. 1994, 116, 4316.

36. Mateos-Timoneda, M. A.; Kerckoffs, J. N. C. A.; CregoCalama, M.; Reinhoudt, D. N. Ditopic Complexation and Release of Neutral Guest Molecules by a Hydrogenbonded "endo-exo" Receptor. Angew. Chem., Int. Ed. 2005 , 44, 3248.

37. Prins, L. J.; De Jong, F.; Timmerman, P.; Reinhoudt, D. N. An Enantiomerically Pure Hydrogen-bonded Assembly. Nature 2000, 408, 181-184.

38. Ranganathan, A.; Pedireddi, V. R.; Rao, C. N. R Hydrothermal Synthesis of Organic Channel Structures: 1:1 Hydrogen-bonded Adducts of Melamine with Cyanuric and Trithiocyanuric Acids. J. Am. Chem. Soc. 1999, 121, 1752.

39. Theobald, J. A.; Oxtoby, N. S.; Phillips, M. A.; Champness, N. R.; Beton, P. H. Controlling Molecular Deposition with Supramolecular Surfaces Assemblies. Nature 2003, 424, 1029-1031.

40. De Feyter, S.; Miura, A.; Yao, S.; Chen, Z.; Würthner, F.; Jonkheijm, P.; Schenning, A. P. H. J.; Meijer, E. W.; De Schryver, F. C. Two-dimensional Self-assembly into Multicomponent Hydrogen-bonded Nanostructures. Nano Lett. 2005, 5, 77-81.

41. Theobald, J. A.; Oxtoby, N. S.; Phillips, M. A.; Champness, N. R.; Beton, P. H.; Dennis, T. J. S. Growth Induced Reordering of Fullerene Clusters Trapped in a Twodimensional Supramolecular Network. Langmuir 2005, 21, 2038.

42. Ludwig, C.; Gompf, B.; Petersen, J.; Stohmaier, R.; Eisenmenger, W. STM Investigations of PTCDA and PTCDI on Graphite and MoS2. A Systematic Study of Epitaxy and STM Image Contrast. Z. Phys. B: Condens. Matter 1994, 93, 365-373.

43. Uder, B.; Ludwig, C.; Petersen, J.; Gompf, B.; Eisenmenger, W. STM Characterization of Organic Molecules on $\mathrm{H}-$ terminated Si(111). Z. Phys. B: Condens. Matter 1995, 97, 389-390.

44. Guillermet, O.; Glachant, A.; Hoarau, J. Y.; Mossoyan, J. C.; Mossoyan, M. Perylene Tetracarboxylic Diimide Ultrathin Film Deposition on Pt(100): a LEED, AES, REELS and STM Study. Surf. Sci. 2004, 548, 129.

45. Swarbrick, J. C.; Ma, J.; Theobald, J. A.; Oxtoby, N. S.; O'Shea, J. N.; Champness, N. R.; Beton, P. H. Square, Hexagonal, and Row Phase of PTCDA and PTCDI on Ag$\mathrm{Si}(111) \sqrt{ } 3 \times \sqrt{ } 3 \mathrm{R} 90^{\circ}$. J. Phys. Chem. B 2005, 109, $12167-12174$.
46. Cañas-Ventura, M. E.; Xiao, W.; Wasserfallen, D.; Müllen, K.; Brune, H.; Barth, J. V.; Fasel, R. Self-assembly of periodic bicomponent wires and ribbons. Angew. Chem. Inter. Ed. 2007, 119, 1846

47. Ruiz-Osés, M.; González-Lakunza, N.; Silanes, I.; Gourdon, A.; Arnau, A.; Ortega, J. E. Self-assembly of Heterogeneous Supramolecular Structures with Uniaxial Anisotropy. J. Phys. Chem. B 2006, 110, 25573-25577.

48. Böhringer, M.; Morgenstern, K.; Schneider, W.-D.; Berndt, R.; Mauri, F.; De Vita, A.; Car, R. Two-dimensional Selfassembly of Supramolecular Clusters and Chains. Phys. Rev. Lett. 1999, 83, 324-327.

49. Böhringer, M.; Morgenstern, K.; Schneider, W.-D.; Berndt, R. Separation of a Racemic Mixture of Two-dimensional Molecular Clusters by Scanning Tuneling Microscopy. Angew. Chem., Int. Ed. 1999, 38, 821-823.

50. Blüm, M.-C.; Cavar, E.; Pivetta, M.; Patthey, F.; Schneider, W.-D. Conservation of Chirality in a Hierarchical Supramolecular Self-assembled Structure with Pentagonal Symmetry. Angew. Chem., Int. Ed. 2005, 44, 5334.

51. Pivetta, M.; Blüm, M.-C.; Patthey, F.; Schneider, W.-D. ThreeDimensional Chirality Transfer in Rubrene Multilayer Islands on Au(111). J. Phys. Chem. B 2009, 113, 4578-4581.

52. Böhringer, M.; Schneider, W.-D.; Berndt, R. Real Space Observation of a Chiral Phase Transition in a TwoDimensional Organic Layer. Angew. Chem., Int. Ed. 2000, 39, 792-795.

53. Parschau, M.; Fasel, R.; Ernst, K. - H. Coverage and Enantiomeric Excess Dependent Enantiomorphism in Two-dimensional Molecular Crystals. Cryst. Growth Des. 2008, 8, 1890-1896.

54. Fasel, R.; Parschau, M.; Ernst, K. -H. Amplification of Chirality in Two-dimensional Enantiomorphous Lattices. Nature 2006, 439, 449-452.

55. Ernst, K. H. Supramolecular Surface Chirality. Top. Curr. Chem. 2006, 265, 209.

56. Chen, T.; Chen, Q.; Pan, G.-B.; Wan, L.-J.; Zhou, Q.-L.; Zhang, R.-B. Linear Dislocation Tunes Chirality: STM Study of Chiral Transition and Amplification in a Molecular Assembly on an HOPG Surface. Chem. Commun. 2009, 2649-2651.

57. Yokoyama, T.; Yokoyama, S.; Kamikado, T.; Okuno, Y.; Mashiko, S. Selective Assembly on a Surface of Supramolecular Aggregates with Controlled Size and Shape. Nature 2001, 413, 619-621.

58. Wintjes, N.; Hornung, J.; Lobo-Checa, J.; Voigt, T.; Samuely, T.; Thilgen, C.; Stöhr, M.; Diederich, F.; Jung, T. A. Supramolecular Synthons on Surfaces: Controlling Dimensionality and Periodicity of Tetraarylporphyrin Assemblies by the Interplay of Cyano and Alkoxy Substituents. Chem.—Eur. J. 2008, 14, 5794-5802.

59. Garcia, M. H.; Mendes, P. J.; Romao Dias, A. Synthesis and Electrochemical Studies of Organometallic Cobalt(III) Complexes with Substituted Benzonitrile Chromophores: NMR Spectroscopic Data as a Probe on the Second-order Non-linear Optical Properties. J. Organomet. Chem. 2005 690, 4063-4071.

60. Enriquez, A. E.; Scott, B. L.; Neu, M. P. Uranium(III)/(IV) Nitrile Adducts Including UI4( $\left.\mathrm{N}^{\prime} \mathrm{CPh}\right) 4$, a Synthetically Useful Uranium(IV) Complex. Inorg. Chem. 2005, 44, 74037413.

61. Siemer, C. J.; VanStipdonk, M. J.; Kahol, P. K.; Eichhorn, D. M. A Coordination Polymer from a Cyanoscorpionate Complex. Polyhedron 2004, 23, 235.

62. Moitsheki, L. J.; Bourne, S. A.; Nassimbeni, L. R. Catenapoly[[bis(thiocyanato- $\mathrm{KN}$ )cobalt(II)]-di- $\mu-2$ aminobenzonitrile-к2N, N']. Acta Crystallogr., Sect. E: Struct. Rep. Online 2005, 61, 2580.

63. Vaughan, O. P. H.; Alavi, A.; Williams, F. J.; Lambert, R. M. Dipole Amplification: A Principle for the Self-Assembly of Asymmetric Monomers on Metal Surfaces. Angew. Chem. 2008, 120, 2456-2460.

64. Cañas-Ventura, M. E.; Xiao, W.; Ruffieux, P.; Rieger, R.; 
Müllen, K.; Brune, H.; Fasel, R. Stabilization of Bimolecular Islands on Ultrathin $\mathrm{NaCl}$ Films by a Vicinal Substrate. Surf. Sci. 2009, 603, 2294-2299.

65. Aït-Mansour, K.; Cañas-Ventura, M. E.; Ruffieux, P.; Jaafar, R.; Bieri, M.; Rieger, R.; Müllen, K.; Fasel, R.; Gröning, O. Strain-relief Pattern as Guide for the Formation of Surfacesupported Bimolecular Nanoribbons. Appl. Phys. Lett. 2009, 95, 143111.

66. Okuno, Y.; Yokoyama, T.; Yokoyama, S.; Kamikado, T.; Mashiko, S. Theoretical Study of Benzonitrile Clusters in the Gas Phase and their Adsorption onto a $\mathrm{Au}(111)$ Surface. J. Am. Chem. Soc. 2002, 124, 7218-7225.

67. Kuck, S.; Chang, S.-H.; Klöckner, J.-P.; Prosenc, M. H.; Hoffmann, G.; Wiesendanger, R. Steering Two-dimensional Molecular Growth via Dipolar Interaction. ChemPhysChem 2009, 10, 2008-2011.

68. Wang, Y.; Hwang, G. S. Origin of Nonlocal Interactions in Adsorption of Polar Molecules $\mathrm{Si}(001)-2 \times 1$. J. Chem. Phys. 2005, 122, 164706.

69. Makoudi, Y.; Arab, M.; Palmino, F.; Duverger, E.; Ramseyer, C.; Picaud, F.; Chrioux, F. A Stable Room-temperature Molecular Assembly of Zwitterionic Organic Dipoles Guided by a Si(111) $-7 \times 7$ Template Effect. Angew. Chem., Int. Ed. 2007, 46, 9287.

70. Baber, A. E.; Jensen, S. C.; Sykes, E. C. H. Dipole-driven Ferroelectric Assembly of Styrene on Au(111). J. Am. Chem. Soc. 2007, 129, 6368-6369.

71. Kim, Y.; Kato, H. S.; Komeda, T.; Kawai, M. STM Investigation of Diffusion Dynamics and 1-D chain Formation of CO Molecules on Pd(110). RIKEN Rev. 2001, 38-32.

72. Yokoyama, T.; Takahashi, T.; Shinozaki, K.; Okamoto, M. Quantitative Analysis of Long-range Interactions Between Adsorbed Dipolar Molecules on Cu(111). Phys. Rev. Lett. 2007, 98, 206102.

73. Dewar, M. J. S.; Zoebisch, E. G.; Healy, E. F.; Stewart, J. J. P. AM1: A New General Purpose Quantum Mechanical Molecular Model. J. Am. Chem. Soc. 1985, 107, 3902.

74. Hill, I. G.; Kahn, A.; dos Santos, D. A.; Cornil, J.; Brédas, J. L. Occupied and Unoccupied electronic levels in organic $\pi$ conjugated molecules: comparison between experiment and theory. Chem. Phys. Lett. 2000, 317, 444.

75. Cornell, W. D.; Cieplak, P.; Bayly, Ch. I.; Gould, I. R.; Merz, K. M.; Ferguson, Jr., D. M.; Spellmeyer, D. C.; Fox, T.; Caldwell, J. W.; Kollman, P. A. A Second Generation Force Field for the Simulation of Proteins, Nucleic Acids, and Organic Molecules. J. Am. Chem. Soc. 1995, 117, 5179.

76. Auwärter, W.; Weber-Bargioni, A.; Riemann, A.; Schiffrin, A.; Gröning, O.; Fasel, R. Self-assembly and Conformation of Tetrapyridyl-porphyrin Molecules on Ag(111). J. Chem. Phys. 2006, 124, 194708 